\title{
Mediastinal silicone lymphadenopathy revealed after thymectomy for autoimmune myasthenia gravis
}

\author{
Sotirios D. Moraitis1, Apostolos C. Agrafiotis1,3, Amalia Kapranou2, Konstantinos Kanakakis1 \\ 1 Department of Thoracic Surgery, Athens Naval and Veterans Hospital, Athens, Greece \\ 2 Department of Pathology, Athens Naval and Veterans Hospital, Athens, Greece \\ 3 Department of Thoracic Surgery, Tenon University Hospital, Paris, France
}

\begin{abstract}
Breast reconstruction is a very popular surgical intervention performed either for cosmetic reasons or after oncological resections. Even though silicone is considered to be an inert material, there are side effects that have been reported, such as silicone lymphadenopathy. In the case reported herein, a silicone lymphadenopathy of the internal mammary and the anterior mediastinal lymph nodes were revealed after a thymectomy for autoimmune myasthenia gravis. Silicone lymphadenopathy should always be part of the differential diagnosis of enlarged lymph nodes, in patients with previous cosmetic or oncoplastic surgery with the use of silicone gel breast implants. Special attention should be paid in case of previous breast cancer in order to rule out metastasis.
\end{abstract}

\section{Introduction}

Breast reconstruction is a very popular surgical intervention performed either for cosmetic reasons (augmentation mammoplasty) or after oncological resections (oncoplastic breast reconstruction) [1]. Silicone gel implants are the most frequently used material for breast

Corresponding author: Dr. Apostolos Agrafiotis, Department of Thoracic Surgery, Tenon University Hospital, 4 rue de la Chine, 75020 Paris, France. E-mail: apostolos.agrafiotis@aphp.fr

Key words: Silicone lymphadenopathy; thymectomy; myasthenia gravis.

Contributions: All the authors contributed equally.

Conflict of interest: The authors have no conflict of interest to declare.

Received for publication: 31 January 2018

Accepted for publication: 9 May 2018

CC Copyright S.D. Moraitis et al., 2018

Tipografia PI-ME Editrice, Italy

Monaldi Archives for Chest Disease 2018; 88:912

doi: 10.4081/monaldi.2018.912

This article is distributed under the terms of the Creative Commons Attribution Noncommercial License (by-nc 4.0) which permits any noncommercial use, distribution, and reproduction in any medium, provided the original author(s) and source are credited. reconstruction. Even though silicone is considered to be an inert material, however, there are side effects that have been reported [1]. One of these is silicone lymphadenopathy, defined as the presence of silicone particles into a lymph node [1-12]. Axillary silicone lymphadenopathy is more frequently described after breast reconstruction with silicone implants; however other lymph node groups can be affected [1-6].

In the case reported herein, a silicone lymphadenopathy of the internal mammary and the anterior mediastinal lymph nodes were revealed after a thymectomy for autoimmune myasthenia gravis.

\section{Case Report}

A 25-year-old female patient was recently diagnosed with an autoimmune myasthenia gravis (MGFA class II symptomatology). The acetylcholine receptor (AchR) serum antibody test was positive. She was treated with pyridostigmine. There was no past medical history apart from an augmentation mammoplasty with silicone gel implants for cosmetic reasons a few years ago. A chest computed tomography (CT) scan was performed in order to detect an eventual thymic pathology. Indeed, there was an anterior mediastinal mass suggestive of a thymoma associated with enlarged internal mammary lymph nodes. There was no sign of capsular retraction or rupture of the breast implants. An 18FDG PET scan showed an hypermetabolic activity of the anterior mediastinal mass and the internal mammary lymph nodes with a standard uptake value $\left(\mathrm{SUV}_{\max }\right)$ of 9 , whereas the $\mathrm{SUV}_{\max }$ of the surrounding mediastinal structures was 1.4 (Figure 1). There was no other locoregional or distant hypermetabolic focus.

The patient was referred by her neurologist to the thoracic surgery department for an elective thymomectomy and thymectomy. Through a median sternotomy, a total thymectomy with bilateral internal mammary lymph node dissection was performed. In fact, there was no thymic tumor but rather a bloc of enlarged lymph nodes in the anterior mediastinal fat adjacent to the thymus gland. The postoperative course was uneventful and the patient was discharged on the $5^{\text {th }}$ postoperative day.

The pathology report confirmed the absence of thymic pathology. On the contrary, the internal mammary lymph nodes and those situated into the perithymic fat presented lesions of granulomatous lymphadenitis associated with lipid vacuoles surrounded by histiocytes (CD68+) and foreign body giant cells (Figures 2 and 3). The histological image was highly suggestive of a silicone lymphadenopathy, taking into consideration the patient's medical history. It is noteworthy that after surgery there was an improvement of the patient's clinical condition, permitting to reduce the pyridostigmine dosage by one half. 


\section{Discussion}

Silicone is the most frequently used material for the manufacture of breast implants, for either cosmetic or oncoplastic surgery [1]. Despite the fact that it is considered to be a biologically inert material, there are complications associated with its use that have been reported [1]. According to Adams et al., local and systematic granulomatous reactions due to silicone implants can affect the breast, lymph nodes, joint capsules, heart, liver and kidneys [2]. Silicone lymphadenopathy implies the migration of silicone particles by tissue macrophages into lymph nodes [1-12]. Axillary nodes are the most frequently affected, but cervical, supraclavicular, intramammary, internal mammary and mediastinal nodes can present silicone lymphadenopathy, as well [1-6]. There are two mechanisms that have been suggested, whether the silicone implants are ruptured or intact $[1,5,6]$. Silicone lymphadenopathy seems to be a rare complication; however, its incidence is not known $[2,6]$. There are case reports and small case series in the literature regarding silicone lymphadenopathy and breast silicone implants [4]. Risk of implant rupture and leakage increases with time after implantation [6]. Zambacos et al. found that the mean time between implantation surgery and implant rupture or leakage was 10.56 years [13]. As stressed by Klang et al., implant ruptures are not clinically apparent and the MRI is the imaging modality of choice for detecting them [5]. According to Collado-Mesa and colleagues the incidence of MRI-diagnosed breast implant ruptures has been estimated to 4.4 ruptures/100 implants per year [6]. In addition, silicone lymphadenopathy can occur after joint replacement by silicone implants [10]. In case of axillary lymphadenopathy, breast cancer or metastasis (in case of previous breast cancer) should be excluded [1,6,11-14]. Moreover, there are case reports showing the occurrence of breast lymphoma in patients with silicone breast implants, and others showing the coexistence of silicone lymphadenopathy and lymphoma in the same lymph node [1,7-9]. As far as silicone mediastinal lymphadenopathy is concerned, few cases are reported in the English literature, illustrating the rarity of this entity $[4,14]$. As far as the opera-

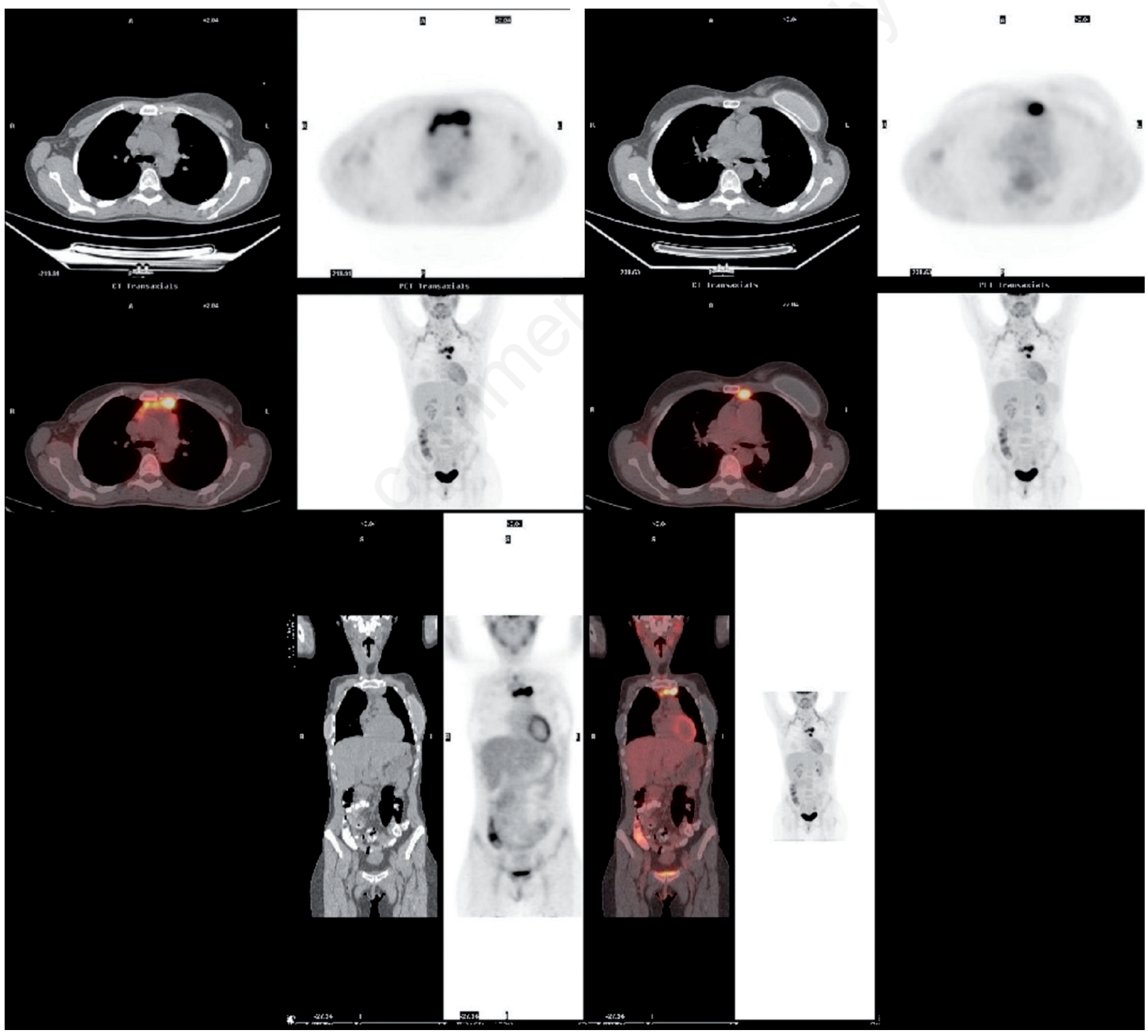

Figure 1. 18FDG PET scan image showing the hypermetabolism of the anterior mediastinal mass and the internal mammary lymph nodes. 

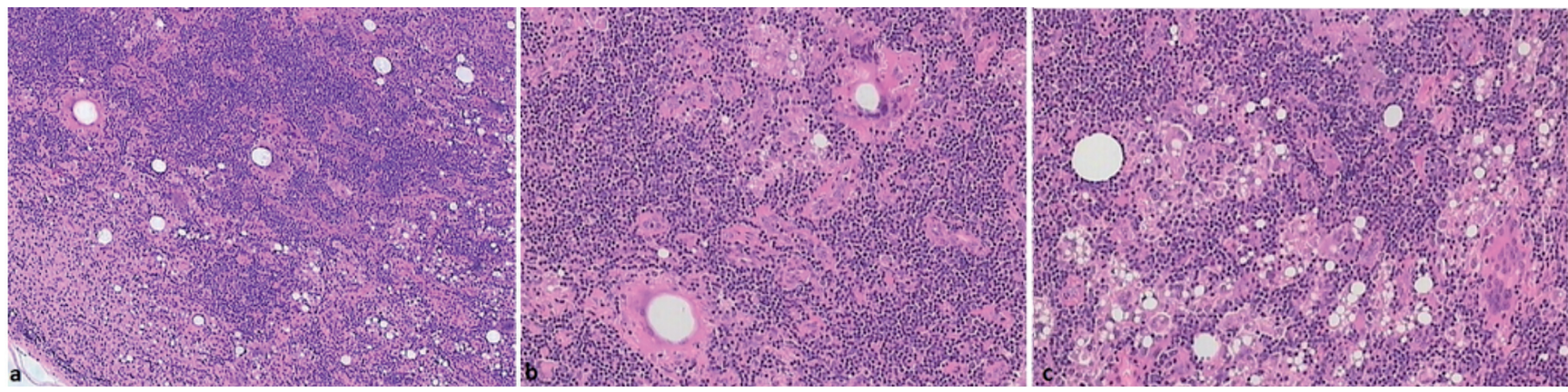

Figure 2. a) Silicone lymphadenopathy (H/E x100); lymph node with lipogranulomatous reaction to lipid: lipid vacuoles in the paracortex surrounded by histiocytes and giant cells, with some of the last ones also containing lipid vacuoles. b,c) Lipid vacuoles in the sinuses surrounded by histiocytes and giant cells (H/E x200).
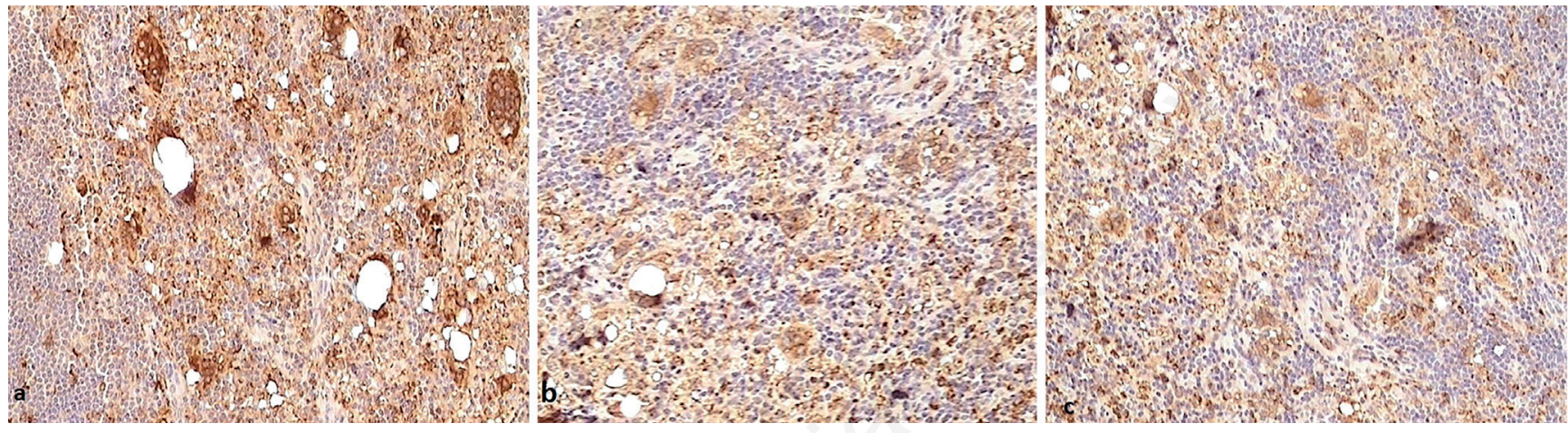

Figure 3. Silicone lymphadenopathy (CD68 x200): CD68 (+) histiocytes and giant cells.

tive access is concerned, a median sternotomy was realized according to a protocol that is implemented in our department. Whenever the ratio between the $\mathrm{SUV}_{\max }$ of an anterior mediastinal mass and the $\mathrm{SUV}_{\text {max }}$ of surrounding mediastinal tissues is $>1$, a median sternotomy is performed.

In the case reported herein, the internal mammary and the anterior mediastinal lymph nodes into the perithymic fat were affected, giving the false image of an anterior mediastinal mass in a patient suffering from an autoimmune myasthenia gravis, who finally presented a clinical improvement after surgery, even in the absence of thymic pathology. Nakahara et al. conducted a retrospective study that showed a long-term improvement after thymectomy in patients with myasthenia gravis even in the group that presented a thymic atrophy [15]. They suggest that, even though the role of thymic atrophy in the pathogenesis of myasthenia gravis remains to be clarified, there is the possibility that immune abnormalities can contribute to the progression of myasthenia gravis patients with thymic atrophy. The same mechanism could be responsible for the clinical outcome of our patient after surgery.

\section{Conclusions}

Silicone lymphadenopathy should always be part of the differential diagnosis of enlarged lymph nodes, not only in the axillary region, in patients that have undergone cosmetic or oncoplastic surgery with the use of silicone gel breast implants. Special attention should be paid in case of previous breast cancer in order to rule out metastasis. Mediastinal silicone lympadenopathy is a rare side effect of breast prostheses and can also mimic other pathologies, especially cancer.

\section{References}

1. Dragoumis DM, Assimaki AS, Vrizas TI, Tsiftsoglou AP. Axillary silicone lymphadenopathy secondary to augmentation mammaplasty. Indian J Plast Surg 2010;43:206-9.

2. Adams ST, Cox J, Rao GS. Axillary silicone lymphadenopathy presenting with a lump and altered sensation in the breast: a case report. J Med Case Rep 2009;3:6442.

3. Borghol K, Gallagher G, Skelly BL. Silicone granuloma from ruptured breast implants as a cause of cervical lymphadenopathy. Ann R Coll Surg Engl 2016;98:e118-20.

4. Maricevich M, Grams J, Abell Aleff P, et al. Mediastinal silicone lymphadenopathy secondary to a ruptured breast implant. Breast J 2011;17:674-5.

5. Klang E, Amitai MM, Raskin S, et al. Association between enlarged axillary lymph nodes and silicone breast implant ruptures seen on magnetic resonance imaging. Isr Med Assoc J 2016;18:719-24.

6. Collado-Mesa F, Yepes M, Doshi P, et al. Contralateral intramammary silicone lymphadenitis in a patient with an intact standard duallumen breast implant in the opposite reconstructed breast. J Radiol Case Rep 2013;7:24-31.

7. Tabatowski K, Elson CE, Johnston WW. Silicone lymphadenopathy in a patient with mammary prosthesis. Fine needle aspiration cytology, histology and analytical electron microscopy. Acta Cytol 1990;34:10-4.

8. Katzin WE, Centeno JA, Feng LJ, et al. Pathology of lymph nodes from patients with breast implants: A histologic and spectroscopic evaluation. Am J Surg Pathol 2005;29:506-11.

9. Van Diest PJ, Beekman WH, Hage JJ. Pathology of silicone leakage from breast implants. J Clin Pathol 1998;51:493-7. 
10. Corrin B. Silicone lymphadenopathy. J Clin Pathol 1982;35:901-2.

11. Soudack M, Yelin A, Simansky D, Ben-Nun A. Fluorodeoxyglucosepositive internal mammary lymph node in breast cancer patients with silicone implants: is it always metastatic cancer? Eur J Cardiothorac Surg 2013;44:79-82.

12. Ganau S, Tortajada L, Rodriguez X, et al. Silicone lymphadenopathy: an unusual cause of internal mammary node enlargement. Breast J 2008;14:502-3.
13. Zambacos GJ, Molnar C, Mandrekas AD. Silicone lymphadenopathy after breast augmentation: case reports, review of the literature, and current thoughts. Aesthetic Plast Surg 2013;37:278-89.

14. Grubstein A, Cohen M, Steinmetz A, Cohen D. Siliconomas mimicking cancer. Clin Imaging 2011;35:228-31.

15. Nakahara K, Nakane S, Nakajima M, Yamashita S, Mori T, Ando Y. Effect of thymectomy for thymic atrophy in myasthenia gravis: A retrospective study on 93 patients. J Neuroimmunol 2017;305:182-185. 